\title{
Relative Survival of Breast Cancer Patients in Iran
}

\author{
Amir Kasaeian ${ }^{2 *}$, Alireza Mosavi-Jarrahi ${ }^{3,4}$, Alireza Abadi ${ }^{5}$, Mahmood \\ Mahmoodi ${ }^{1}$, Yadollah Mehrabi ${ }^{6}$, Kazem Mohammad ${ }^{1}$, Mohammad Reza \\ Eshraghian ${ }^{1}$, Ali Zare ${ }^{1}$
}

\begin{abstract}
Background: The survival rate reflecting prognosis of breast cancer patients is usually estimated based on crude survival methods such as observed and cause-specific. In situations where data are based on population-cancer registries, this method may produce biased estimations. This study therefore aimed to estimate the net survival of breast cancer based on relative survival. Materials and Methods: Data for 622 breast cancer patients diagnosed at the Iran Cancer Institute during 1990-95 and tracked till the end of 2000 were analyzed. For estimation of relative survival, Ederer's second method and SAS (9.1) and STATA (11) software were used. Results: Threeyear relative survivals of $85 \%, 90 \%, 80 \%$ and $67 \%$ were observed for age groups $15-44,55-59,60-74$, and $75+$ years-old, respectively. A relative survival of approximately one was observed for two subsequent years for age-group 45-59 years-old. A value greater than one for two subsequent years of follow-up was observed in the age-group 60-74 years-old. Conclusions: Tracking the diagnosis of breast cancer, the relative survival decreases as we go to higher age-groups. It is also perceived that through follow-up, relative survival first decreased and then increased a little. The statistical cure point is acceptable for age group 45-59 years-old while for age-groups 15-44 and 60-74 years old is a sign of low quality data for some follow-up intervals.
\end{abstract}

Keywords: Breast cancer - cause-specific survival - net survival - relative survival - cause-specific survival

Asian Pac J Cancer Prev, 16 (14), 5853-5858

\section{Introduction}

For cancer, the survival of the patient is accepted as the main criterion of the cancer-control and measuring the treatment effect (Ederer et al., 1961; Parkin, 2008). The survival rate, that is the ratio of the patients who survive in a specific period of time, is a simple concept, but there are various methods for estimating it (Ngoan et al., 2006; Ramadurai and Ponnuraja, 2011; Rahimzadeh et al., 2014; Zare et al., 2014). To analyze the survival of the cancer patients in a specific period of time and based on the data derived from population-based cancer registries, the ratio of relative survival as an estimate of the net survival, has been a more eligible criterion in comparison with observed and cause-specific survival (Kristinsson et al., 2007; Hinchliffe et al., 2012; Dickman et al., 2013; Zare, 2013; Zeng et al., 2014). A major benefit of this measure is that information on the cause of death is not required, thus it circumvents complications with the inaccuracy, incorrectness and non-availability of death certificates and problems due to complicated cures generally codifying and filing cause of death (Erhardt, 1958; Percy et al.,
1981; Utada et al., 2012). One the other hand, most of the cancer patients are middle-aged people who are subjected to other causes of death and such risks should be modified in the survival analysis (Parkin et al., 2005; Zare, 2013; Siegel et al., 2014). The relative survival, which is defined as the ratio between the rate of observed survival and expected survival, carries out the necessary modification for the expected death rate from other reasons related to the disease under study, without any need to cause of death data (Ederer et al., 1961; Utada et al., 2012). In fact, the ratio of the relative survival is a real criterion of the deceased patients who died of the direct or indirect consequences of the disease under study in a population. In such situations in which the survival of the patients is compared with the general condition of the patients, the results indicate the whole condition of the population and it is a reflection of the effects of cancer-control activities in health system of the population (Bell et al., 1995; Parkin, 2008). This research aimed to estimate the relative survival of breast cancer patients who referred to Imam Khomeini Cancer Institute during 1990-95.

${ }^{1}$ Department of Epidemiology and Biostatistics, School of Public Health, ${ }^{2}$ Non-Communicable Diseases Research Center, Endocrinology and Metabolism Population Sciences Institute, ${ }^{4}$ The Cancer Research Center of the Cancer Institute, Tehran University of Medical Sciences, ${ }^{3}$ Department Social Medicine, ${ }^{5}$ Department of Community and Health, School of Medicine, ${ }^{6}$ Department of Epidemiology, School of Public Health, Shahid Beheshti University of Medical Sciences, Tehran, Iran *For correspondence: amir_kasaeian@yahoo.com 


\section{Materials and Methods}

The present study followed up a cohort of 662 patients who were eligible for the conditions of this research. These conditions are: A) the females suffering from breast cancer whose disease was definitely diagnosed through pathological tests, B) patients who referred to Imam Khomeini Cancer Institute during 1990-95, C) if the age of the patients was recorded in diagnosis stage and entering-into-study time, D) if the patients' vital conditions, including survival, death, or lack of follow-up were definite, E) if the survival period or the date of entering into and leaving the study were definite. From among 662 patients 349 were censored, 312 died in the above-mentioned period, and one has not been followed up. Among the 312 deaths, 245 were died due to cancer and 67 were died because of other reasons. This study employed Ederer's second method, among the available methods for computing the rate of the relative survival and decision making about the cancer-patients' treatment, which according to the researches is more effective and relevant in comparison with the other two available methods (Ederer and Heise; Ederer et al., 1961; Hakulinen, 1982). Ederer and Heise suggested a method for estimating the expected survival which computes the actual heterogeneous follow-up times. The so-called Ederer's second method is a good estimator for the ratio of the expected survival of the especial interval (Ederer et al., 1961; Perme et al., 2012). There are other methods to estimate the expected survival which are known as Ederer's first method and Hakulinen's method. Because of not computing the heterogeneity of follow-up times, these methods are not appropriate for the populationbased analyses and if employed, will produce biased estimation (usually over estimation) of relative survival (Dickman and Coviello, 2015). Needing the information of follow-up times of all patients is another disadvantage of Hakulinen's method (Ederer and Heise; Hakulinen, 1982). The Life Table was also used to estimate the amounts of survival because the Life Table was a suitable tool to delineate patients' survival in a long-period of follow-up (Coviello, 2015). To estimate the relative survival, the estimation of the expected survival in the comparative group of the general population who are not supposed to have cancer in action, is required. The estimation of the expected survival is adjusted with age, sex and the calendar-time of diagnosis (Berkson and Gage, 1950). Then the population-based survival ratios are estimated for each adjusted group (Coleman et al., 1999; Quinn et al., 2001). These estimations are based on annual death probability tables in general population. In this study, Finland, for having better health and hygiene conditions, was singled out as a standard source to estimate the expected survival and to compare that with the observed survival of annual death information. Ederer et al. (1961) showed that although these tables encompass the understudy-cancer-based deaths, they have no effect on the estimation of the expected survival in action (Ederer et al., 1961). So, in this way relative survival calculated by dividing the observed survival of the patients by the estimation of the expected survival which was analyzed and discussed in the following. SAS software (version 9) and Stata (version 11) was used to estimate the expected and relative survivals.

\section{Results}

Table 1 reports the observed, expected, and relative survivals according to the age groups. Based on table 1, the annual relative survival in the ninth year of follow-up for the age group of 15-44 is 0.89647 which demonstrates annual death rate as 10.3 percents for the cancer patients in this age group. Since all the estimations are faced with random error, with the effect which is even more in the earlier intervals, so the overall pattern should be considered in relative survivals which is possible with drawing a diagram. The cumulative relative survival is interpreted in time $t$ as a ratio of the patients who survive at least $\mathrm{t}$ years in the hypothetical situation in which the under-study cancer was the only reason for their death. The cumulative relative survival is almost applied after a five-year follow-up as a sole criterion of the experience from population survival. This criterion which is called the five-year cumulative relative survival is interpreted as the ratio of those patients who survived five years after follow-up, supposing that the cancer under study was the sole reason of their deaths. For example, the five-year cumulative relative survival for the patients aged between 15-44 suffering from breast cancer, which is derived from the multiplication of the ratios of the relative survival of

Table 1. Life Tables of Observed, Expected and Relative Survival of Breast Cancer Patients during the Calendar Period 1990-1995 Following up till the End of 2000

\begin{tabular}{|c|c|c|c|c|c|c|c|c|c|c|c|c|}
\hline \multirow{2}{*}{$\begin{array}{l}\text { Fallow } \\
\text { up Year }\end{array}$} & \multicolumn{4}{|c|}{ Observed survival } & \multicolumn{4}{|c|}{ Expected survival } & \multicolumn{4}{|c|}{ Relative survival } \\
\hline & r $15-44$ & $45-49$ & $60-74$ & $75+$ & $15-44$ & $45-49$ & $60-74$ & $75+$ & $15-44$ & $45-49$ & $60-74$ & $75+$ \\
\hline $0-1$ & 0.96137 & 0.94531 & 0.94483 & 0.625 & 0.99899 & 0.99691 & 0.98618 & 0.91736 & 0.96235 & 0.94825 & 0.95806 & 0.6813 \\
\hline 2-Jan & 0.88636 & 0.94839 & 0.84444 & 0.6 & 0.99891 & 0.99673 & 0.98466 & 0.93626 & 0.88733 & 0.9505 & 0.8676 & 0.64085 \\
\hline 3-Feb & 0.85263 & 0.8988 & 0.78475 & 0.625 & 0.99882 & 0.99655 & 0.98317 & 0.9264 & 0.85363 & 0.9014 & 0.79819 & 0.67465 \\
\hline 4-Mar & 0.94872 & 0.85795 & 0.76471 & 0 & 0.9987 & 0.99639 & 0.98182 & 0.90974 & 0.94995 & 0.86106 & 0.77886 & 0 \\
\hline 5-Apr & 0.92254 & 0.92029 & 0.86885 & 0.99862 & 0.99623 & 0.98063 & 0.92381 & 0.92377 & 0.88601 & & & \\
\hline 6-May & 0.92523 & 0.85859 & 0.82609 & 0.99847 & 0.99608 & 0.97833 & 0.92665 & 0.86196 & 0.84438 & & & \\
\hline 7-Jun & 0.89552 & 0.91919 & 0.92 & 0.9982 & 0.99561 & 0.98274 & 0.89714 & 0.92324 & 0.93616 & & & \\
\hline 8-Jul & 0.96923 & 1 & 1 & 0.99823 & 0.99474 & 0.97832 & 0.97095 & 1.00529 & 1.02216 & & & \\
\hline 9-Aug & 0.89474 & 1 & 1 & 0.99807 & 0.99425 & 0.98623 & 0.89647 & 1.00579 & 1.01396 & & & \\
\hline 10-Sep & 1 & & & 0.99757 & & & & & 1.00244 & & & \\
\hline
\end{tabular}




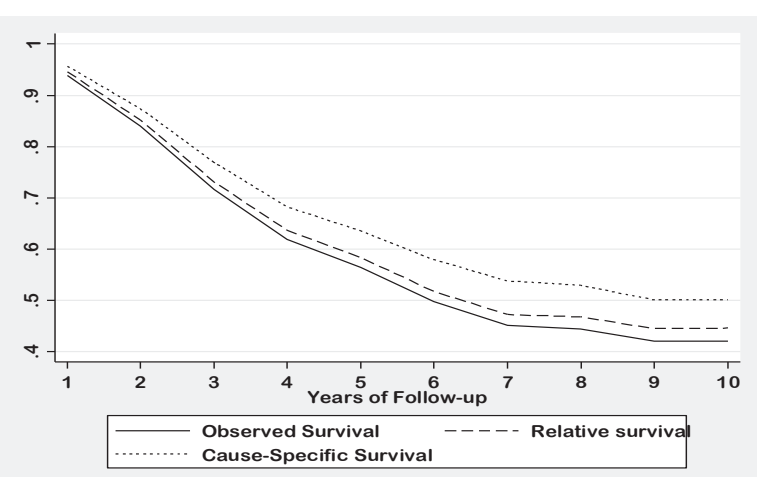

Figure 1. Observed, Cause-specific and Relative (Cumulative) Survival of Breast Cancer Patients during the Study Time

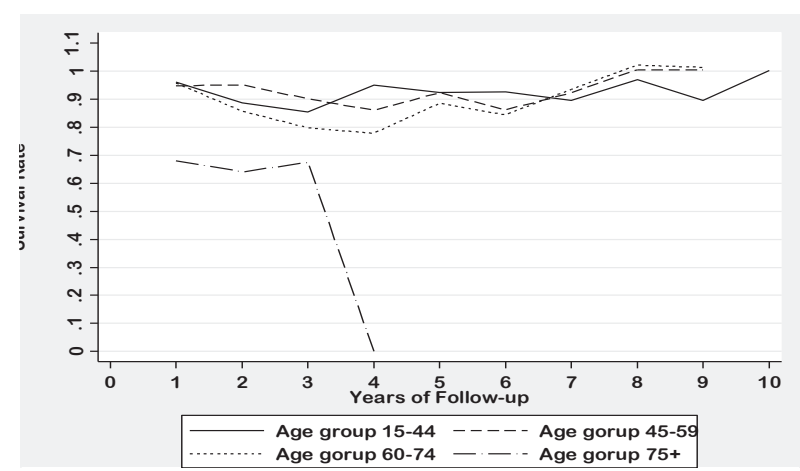

Figure 2. Relative Survial of Breast Cancer Patients by Age Groups

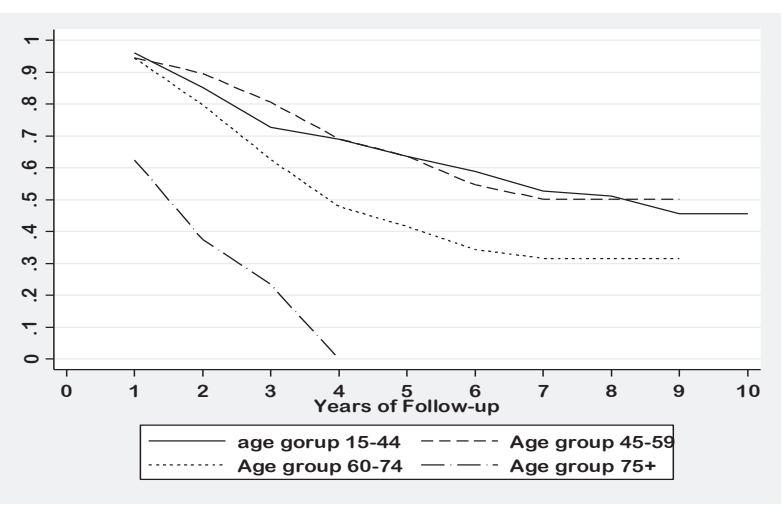

Figure 3. Cumulative Relative Survival of Breast Cancer Patients by Age

the interval-specific up to the fifth year according to Table 1 , was 0.63970 which estimates the survival of these patients up to five years after diagnosis. Sometimes, the estimation of the relative survival in a follow-up period equals one which means that the death rate of the patients equals the death rate of a similar group from the general population. In other words, there is no death as a result of cancer, and if this amount of one retains in the next several follow-up periods, these patients will statistically regarded as 'cured' (Cutler and Axtell, 1963; Kerr et al., 1998). On the other hand, as table 1 shows, sometimes the estimation of the relative survival during a period becomes greater than one which means that the cancer death rate in the observed group is less than the comparable group in the general population and this shows the low quality

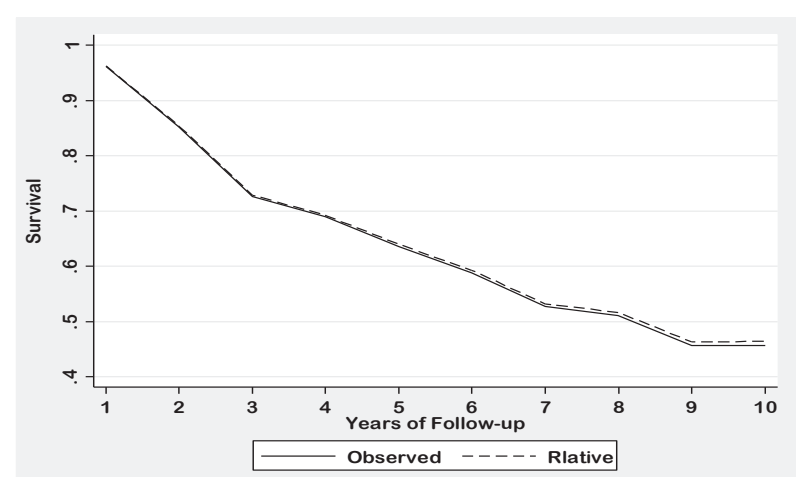

Figure 4. Cumulative Observed and Relative Survival of Breast Cancer Patients

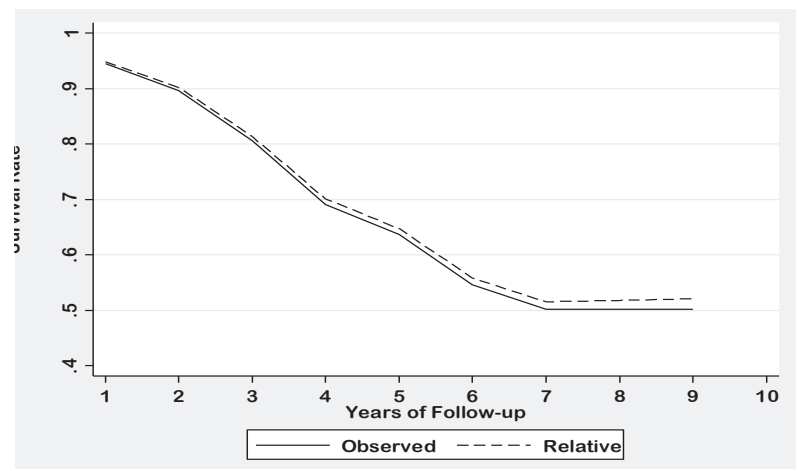

Figure 5. Cumulative Observed and Relative Survival of Breast Cancer Patients in 45-59 Age-group

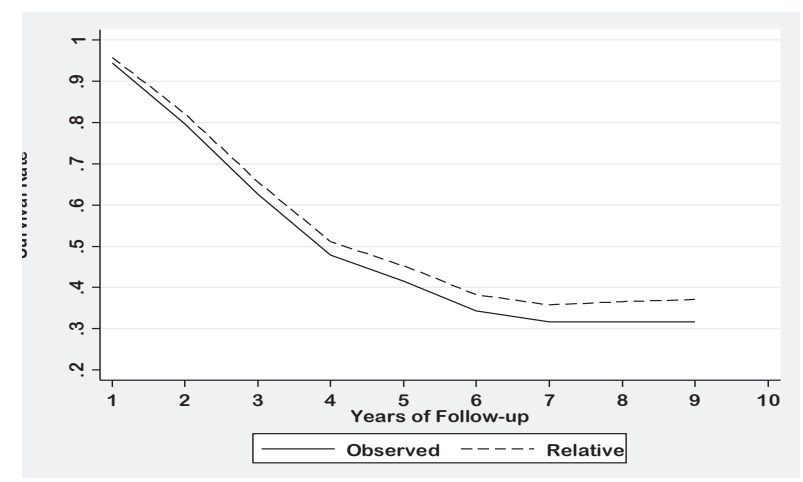

Figure 6. Cumulative Observed and Relative Survival of Breast Cancer Patents in 60-74 Age-group

of the follow-ups (and this reveals the lack of regular follow-up). In table 1, the first ratio of the relative survival is approximately one for the eighth year of the follow-up which means that no breast-cancer-related death has been reported in patients aged 45-95 in this interval. Thus it could be accepted that the patients who survived seven years of follow-up, since the diagnosis of the breast cancer, are statistically regarded as 'cured'.

The cause-specific survival that only deaths resulting from the under-study-disease are regarded as events in its estimation has been compared with observed and relative survivals in figure 1. As it is seen, the difference between the cause-specific and relative survivals is considerable in the estimation of the net survival. It should be notified here that the estimation of the cause-specific survival has its own problems including the lack of making proper diagnosis about the cause of death and reliable codified 


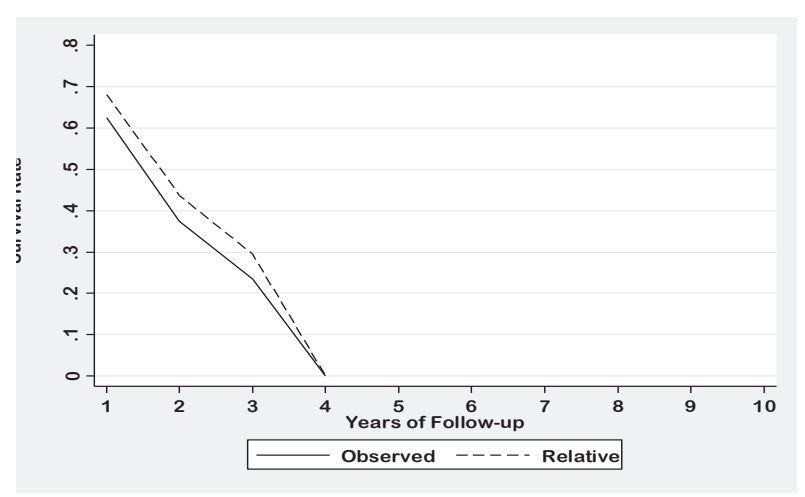

Figure 7. Cumulative Observed and Relative Survival of Breast Cancer Patients in 75+ Age-group

information. This figure has been drawn for the whole information of all patients. The diagrams of the specificinterval relative survival which are statistically utilized to make sure about the treatment are seen in Figure 2. As it is shown no statistical cure point is acceptable for any agegroup except the statistical cure point for the eighth year of follow-up for the age-group of 45-59. Figure 3 shows the ratios of the cumulative relative survival. These diagrams are employed to show the cumulative survival probability to a specific time point but they are not appropriate for determining excess deaths caused by cancer.

As it is seen, the survival experience in the first (15$44)$ and second (45-59) age groups are similar and for the two other groups patients' survival decreases more rapidly their age increases.

Figures 4-7 show the comparison between the estimation of the cumulative relative survival and the cumulative observed survival for different age groups. These figures show that with the increase of the patients' age groups, the discrepancy in survival becomes greater which is a result of the experience of more deaths caused by cancer in greater ages.

\section{Discussion}

In addition to interpreting the results of the study in this part the results will also be compared with results of other studies. It should be pointed out that since Finland population was used in the estimation of the expected survival, the comparison of the present results would not be relevant enough. In the process of finding a population Life Table in Iran, as it was expected, a unique, complete, and reliable Life Table does not exist. Although considerable efforts have been made in this area, such as research projects, MA theses, and $\mathrm{PhD}$ dissertations, there is no unanimous verdict and each of these efforts has put forward tables with a special method in which superficial similarities exist. Logically speaking, in such situation it was ascertained that a substitute Life Table could be replaced; a table whose scientific method and accurate data collection were reliable. To do so, it was consented to use a Life Table of a developed country which has population stability and in which obtaining information is accessible. So the Life Table of Finland was employed. Using Finland's Life Table the researchers could, at least, measure the survival chance of the patients from cancer vis-à-vis a population who was living in a high standard, healthy and hygienic society. On the other hand, one of the objectives of the present research was to introduce the relative survival method in Iran which has not been used yet whereas this method is generally used in cancer registries all over the world to report on cancer status in population (Coleman et al., 2003; Parkin et al., 2005; Jemal et al., 2011; Utada et al., 2012).

According to the findings, the ratio of the observed and relative survivals has decreased in the early followup years and has increased in the terminating follow-up years. This situation is caused because of not observing death among the cancer patients in the terminating followup years. The increase in relative survival and reaching the value one are because of the fact that the observed deaths among the patients have corresponded the deaths among the general population. This situation is statistically indicative of a 'cure' for the cancer patients. According to the findings, the cure point is acceptable after the seventh year of follow-up for the patients aged 45-59 because this value approximately equals one. The interpretation of the cure point for this age group is that if the patients aged 45-59 survive up to the eighth year of follow-up, they will recover. Such situation will occur for the patients aged 15-44 after ninth year of follow-up. Medically speaking, a patient is regarded as 'cured' if no symptom of the disease is detected anymore, whereas statistically speaking, 'cure' occurs in groups not individuals. Sometimes, the fiveyear relative survival, which is used as a criterion report of the patients' survival in cancer registries, is wrongly interpreted as the ratio for the cured patients. Such an interpretation is required to get to the cure point before the fifth year of follow-up which does not normally happen. This study confirms this fact. Sometimes relative survival is estimated greater than one. This situation occurs where the cancer under study is more prevalent in higher socioeconomic groups or where healthy worker effect occurs. The healthy worker effect, sometimes, happens amongst the cancer patients with a low rate of fatality such as skin cancer. The relative survival for these patients is obtained greater than one because few of the patients die due to cancer and also the survivors have a lower rate of mortality from other causes due to greater use of health services.

Of course, it is extremely complicated to determine the exact causal mechanism of this kind. For example, the patients who benefit from health checks and consequently have a better health, are diagnosed at the early stages and the regular contact with the health system after disease diagnosis has a positive effect on their general health. But the most probable reason for the estimation of relative survivals whose amounts are greater than one is the low quality of the follow-up. If the deaths among the cancer patients are not registered, the survivals among patients will be estimated greater than the real amount, and this will lead to relative survival estimations greater than one. That is why the study of the specific-interval relative survival is regarded as a useful method to evaluate the quality of the patients' follow-ups. Such situation was observed for the age group of 60-74 after the seventh year of follow-up. The comparison between the diagrams of the survival ratios for 
different age groups demonstrated that the survival status for the age group whose patients aged more than 75 was completely different from the other three groups which is a natural issue because disease-related deaths have a direct relation with age increasing. The results from the diagramcomparison of the observed and relative survivals showed that the more increase in age, the greater difference between the observed and relative survivals. In fact, as age increases the observed survival of the cancer patients is estimated lower than its real value. This is natural because the risk of death due to other causes is greater for older people and the observed survival regards all causes of deaths as outcome of interest. Whereas for younger patients the observed survival, because of negligibility for the other causes of deaths, is similar to the two other rates. Moreover, the cancer survival in adults, even after adjusting other causes of deaths, is heavily age-dependent (Sant et al., 2003; Sarfati et al., 2010). Such problem does not exist in the estimation of relative survival and, thus, is considered as an estimation of net survival. The fiveyear relative survival for the 45-59 age group is greater in comparison with other age groups which is supported by the results of this study (Coleman et al., 2003). The results of the current study accentuate the suitability of relative survival as measurement to estimate the net survival of the cancer patients.

However, the relative survival study on breast cancer throughout the world pinpoints crucial points. A Concord population-based study which was conducted as a historical cohort on 1.9 million subjects all over the world, demonstrated that the five-year relative survival of breast, colorectal and prostate cancers are generally high in North America, Australia, Japan, the north, the south, and the west of Europe and low in countries like Algeria, Brazil, and Eastern Europe. The results of this study which encompasses about $42 \%$ of American population shows that cancer survival in male and female blacks is significantly lower than in male and female whites (Coleman et al., 2008).

The results of another study conducted on eight major cancers in Europe revealed that the five-year age-adjusted relative survival mean for breast cancer in central northern European countries (except Denmark) has had the highest value, in southern Europe average, low in England, and the lowest and the worst amount in eastern Europe. In this study the cancer patients entered into the research during 1995-99 and they were followed up to the end of 2003. The findings disclosed that the five-year relative survival was about $83 \%$ in Finland and Switzerland, about $82 \%$ in France and Italy, about $81 \%$ in the Netherlands, and about $77 \%$ in England and Denmark. The findings also showed that the five-year relative survival in 95-99 period vis-àvis 90-94 period in Poland, Ireland, and Czech Republic were with increasing amounts of $12.1 \%, 8.9 \%$, and $7.9 \%$ respectively (Berrino et al., 2007). Sant et al. demonstrated as a part of the comprehensive Eurocare study that the mean of the five-year regional and age-adjusted relative survival for the breast-cancer patients diagnosed in 9599 was about $79 \%$ whereas the same index for $90-94$ has been obtained about $76 \%$. In this study, Poland, Czech Republic, and Slovenia had the lowest rate of cancer (about 73\% and lower) and Ireland, Switzerland, and Italy obtained the highest rate for cancer survival (Sant et al., 2009).

According to the present study the following suggestions are made: $i)$ Enhancing the populationbased cancer registries in country by collecting and storing general information about all kinds of cancers and following them up more accurately and precisely. ii) Employing the relative survival to report on the status of different kinds of cancer and chronic diseases such as heart disease in population. iii) Regression modeling of relative survival and estimating the effect of factors like age, diagnosis period, and disease stage on the survival rate of the cancer patient.

\section{Acknowledgements}

We hereby express our deepest gratitude to the managers and staff of the cancer research center of Cancer Institute of Imam Khomeini medical complex that made this research come to fruition by providing us with the necessary and available data.

\section{References}

Bell C, Lawrence G, Pheby D, Smith J, Coleman M(1995). The role of cancer registries. Clinical Oncol, 7, 143-144.

Berkson J and Gage RP (1950) Calculation of survival rates for cancer. Proceedings of the staff meetings. Mayo Clinic, 25, 270-286.

Berrino F, De Angelis R, Sant M, et al (2007). Survival for eight major cancers and all cancers combined for European adults diagnosed in 1995-99: results of the EUROCARE-4 study. Lancet Oncol, 8, 773-783.

Coleman M, Gatta G, Verdecchia A, et al (2003). EUROCARE-3 summary: cancer survival in Europe at the end of the 20th century. Ann Oncol, 14, 128-149.

Coleman MP, Babb P, Damiecki P, et al (1999) Cancer survival trends in England and Wales, 1971-1995: deprivation and NHS region: Stationery Office London.

Coleman MP, Quaresma M, Berrino F, et al (2008) Cancer survival in five continents: a worldwide population-based study (CONCORD). Lancet Oncol, 9, 730-756.

Coviello E, P. W. Dickman, K. Seppa, and A. Pokhrel (2015) Estimating net survival using a life-table approach. Stata $J, 15,173-85$.

Cutler SJ and Axtell LM (1963) Partitioning of a patient population with respect to different mortality risks. $J \mathrm{Am}$ Statistical Associat, 58, 701-712.

Dickman P and Coviello E (2015) Estimating and modeling relative survival. Stata $J, \mathbf{1 5}, 186-215$.

Dickman PW, Lambert PC, Coviello E, Rutherford MJ (2013) Estimating net survival in population-based cancer studies. Int J Cancer, 133, 519-21.

Ederer F, Axtell LM, Cutler SJ (1961) The relative survival rate: a statistical methodology. National Cancer Institute Monograph, 6, 101-121.

Ederer F and Heise H Instructions to IBM 650 Programmers in Processing Survival Computations; 1959. National Cancer Institute, Bethesda (MD).

Erhardt CL (1958) What Is The Cause Of Death? J Am Med Associat, 168, 161-8.

Hakulinen T (1982) Cancer survival corrected for heterogeneity in patient withdrawal. Biometrics, 38, 933-42. 
Amir Kasaeian et al

Hinchliffe S, Rutherford M, Crowther M, Nelson C and Lambert P (2012) Should relative survival be used with lung cancer dataandquest. British J Cancer, 106, 1854-1859.

Jemal A, Bray F, Center MM, Ferlay J, Ward E and Forman D (2011) Global cancer statistics. CA Cancer J Clin, 61, 69-90.

Kerr G, Kunkler I, Langlands A and Rodgert A (1998) (In) curability of breast cancer: a 30-year report of a series of 3933 cases. Breast, 7, 90-94.

Kristinsson SY, Landgren O, Dickman PW, Derolf ÅR and Björkholm M (2007) Patterns of survival in multiple myeloma: a population-based study of patients diagnosed in Sweden from 1973 to 2003. J Clin Oncol, 25, 1993-9.

Ngoan IT, Long TT, Lu NT, Hang L (2006) Population-based cancer survival in sites in Viet Nam. Asian Pac J Cancer Prev, 8, 539-42.

Parkin DM (2008). The role of cancer registries in cancer control. Int J Clin Oncol, 13, 102-11.

Parkin DM, Bray F, Ferlay J, Pisani P (2005) Global cancer statistics, 2002. CA Cancer J Clin, 55, 74-108.

Percy C, Stanek $3^{\text {rd }}$ E, Gloeckler L (1981) Accuracy of cancer death certificates and its effect on cancer mortality statistics. Am J Public Health, 71, 242-250.

Perme MP, Stare J, Esteve J (2012) On estimation in relative survival. Biometrics, 68, 113-20.

Quinn M, Babb P, Brock A, Kirby L, Jones J (2001) Cancer trends in England and Wales, 1950-1999: Stationery Office.

Rahimzadeh M, Baghestani AR, Gohari MR, Pourhoseingholi MA (2014) Estimation of the cure rate in Iranian breast cancer patients. Asian Pac J Cancer Prev, 15, 4839-42.

Ramadurai M, Ponnuraja C (2011). Non-parametric estimation of the survival probability of children affected by TB meningitis. Int Refereed Res J, 2, 216-27.

Sant M, Aareleid T, Berrino F, et al (2003) EUROCARE-3: survival of cancer patients diagnosed 1990-94-results and commentary. Ann Oncol, 14, 61-118.

Sant M,Allemani C, Santaquilani M, et al (2009). EUROCARE-4. Survival of cancer patients diagnosed in 1995-1999. Results and commentary. Eur J Cancer, 45, 931-91.

Sarfati D, Blakely T and Pearce N (2010). Measuring cancer survival in populations: relative survival vs cancer-specific survival. Int J Epidemiol, 39, 598-610

Siegel R, Ma J, Zou Z, Jemal A (2014). Cancer statistics, 2014. CA Cancer J Clin, 64, 9-29.

Utada M, Ohno Y, Shimizu S, Hori M, Soda M (2012). Comparison between overall, cause-specific, and relative survival rates based on data from a population-based cancer registry. Asian Pac J Cancer Prev, 13, 5681-5.

Zare A, Mahmoodi M, Mohammad K, et al (2014). A Comparison between Kaplan-Meier and Weighted KaplanMeier Methods of Five-Year Survival Estimation of Patients with Gastric Cancer. Acta Medica Iranica, 52, 764-7.

Zare A, Mahmoodi, M. (2013). Modified kaplan-meier estimator based on competing risks for heavy censoring data. Int $J$ Statist Med Res, 2, 297-304.

Zeng H, Zheng R, Guo Y, et al (2015). Cancer survival in China, 2003-2005: A population-based study. Int J Cancer, 136, 1921-30. 